\title{
The optimality principle for second-order discrete and discrete-approximate inclusions
}

\author{
Sevilay Demir Sağlam* \\ Department of Mathematics, University of Istanbul,Turkey \\ sevilay.demir@istanbul.edu.tr
}

\section{ARTICLE INFO}

\section{Article History:}

Received 14 December 2020

Accepted 21 February 2021

Available ** May 2021

\section{Keywords:}

Discrete inclusion

Approximation

Optimality condition

Locally adjoint mapping

AMS Classification 2010:

49K15; 49M25; $49553 ; 37 M 15$

\begin{abstract}
This paper deals with the necessary and sufficient conditions of optimality for the Mayer problem of second-order discrete and discrete-approximate inclusions. The main problem is to establish the approximation of second-order viability problems for differential inclusions with endpoint constraints. Thus, as a supplementary problem, we study the discrete approximation problem and give the optimality conditions incorporating the Euler-Lagrange inclusions and distinctive transversality conditions. Locally adjoint mappings (LAM) and equivalence theorems are the fundamental principles of achieving these optimal conditions, one of the most characteristic properties of such approaches with second-order differential inclusions that are specific to the existence of LAMs equivalence relations. Also, a discrete linear model and an example of second-order discrete inclusions in which a set-valued mapping is described by a nonlinear inequality show the applications of these results.
\end{abstract}

(c) $)$ EY

\section{Introduction}

Optimal control problems with discrete and differential inclusions are increasingly studied in mathematical theory [1,2]. Also, discrete and continuous-time processes have wide applications in the field of mathematical economics and the problems of control dynamic system optimization 3 6]. Thus, the optimal control problems described by discrete inclusions with endpoint constraints and approximation play a very significant role in both theory and applications of control theory [7, 8].

Second-order discrete and differential inclusions have been studied by many authors when the setvalued mapping is both convex and nonconvex valued (see [9-11] and references therein).

Auslender and Mechler 12 establish necessary and sufficient conditions to ensure the existence of solutions to the second-order differential inclusions with state constraints via interior tangent sets.

\footnotetext{
*Corresponding author
}

An approximation to a linear differential inclusion using $\mathrm{N}$-stage single step discrete inclusions is described in the paper [13]. The result is applied to the discretization of control constrained optimal control problems in the second-order and the use of dynamic programming for approximate feedback design.

The paper 14 deals with the discrete approximations of nonconvex differential inclusions in Hilbert spaces and dynamic optimization-optimal control problems concerning such differential inclusions.

Agarwal and O'Regan [15] present new fixedpoint theorems for weakly sequentially upper semicontinuous maps. These results are used to establish existing principles for second-order differential equations and inclusions.

The optimal control of discrete-time systems are given in the book of Boltyanskii [16. That book explores some results from the classical control 
point of view for a linear, time-invariant, discretetime, optimal control system with infinite-time case.

In general, several studies on the differential inclusions of the second-order are devoted to problems of existence and viability. In the case where the multifunction is upper semicontinuous and has compact convex values, Haddad and Yarou [17] provided the first viability result for secondorder differential inclusions. The Cauchy problem for the infinite-dimensional case and second-order differential inclusions are considered in that paper.

As is pointed out in the paper [18, Marco and Murillo analyzed the existence of Lyapunov functions for second-order differential inclusions by using the methods of the viability theory. A necessary assumption on the initial states and sufficient conditions are obtained for the existence of local and global Lyapunov functions.

Lupulescu [19] demonstrated the existence of viable solutions for autonomous second-order functional-differential inclusions in the case where the multifunction defining the inclusion is upper semicontinuous, compact valued, and contained in the subdifferential of a proper lower semicontinuous convex function.

Due to the higher-order derivatives and their discrete analogs, the problems accompanied by the higher-order discrete and differential inclusions are more complicated. A convenient procedure for eliminating this complication in optimal control theory involving higher-order derivatives is a formal reduction of the problems by substitution to the system of first-order differential inclusions or equations. But in practice, returning to the higher-order problem and expressing the arising optimality conditions in terms of the original problem data, in general, is very difficult. Although the construction of adjoint inclusions and transversality conditions is more complicated, Mahmudov formulates the conditions of optimality for the optimal control problem of higher-order differential inclusions with functional constraints in the paper [20].

The principle approach we use is that of locally adjoint mapping $(L A M)$, which facilitates obtaining necessary and sufficient conditions for all types of discrete and differential inclusions. Optimization of various forms of discrete inclusions can be reduced to finite-dimensional problems of mathematical programming, namely, to the minimization of functions on the intersection of a finite number of sets.

We use difference approximations of ordinary derivatives and grid functions on a uniform grid to approximate differential inclusions and to derive necessary and sufficient conditions of optimality for discrete-approximation problems. It turns out that this requires some particular equivalence theorems of a $L A M$, which arise in discrete and discrete-approximation problems.

One of the central objects of this paper is the relationship between continuous and discrete systems. By using particular equivalence theorems of the $L A M$, which play a significant role in the following investigations and without which few necessary or sufficient conditions would be obtained, the transition to the optimal conditions for discrete-approximation problems from their discrete counterparts is realized. The point argument is that discrete and discrete-approximation problems naturally are described by different setvalued mappings (say $F$ and $G$, respectively). Therefore, we have to express the LAM $G^{*}$ by $F^{*}$ to formulate the optimality conditions for each discrete and discrete-approximation problem associated with the continuous problem.

The rest of this paper is organized as follows.

For the convenience of the reader, the necessary facts and supplementary results from the book of Mahmudov [1] are summarized in Section 2. In particular, the Hamiltonian function, argmaximum set of a set-valued mapping, and the locallyadjoint mapping are introduced and the viability problems for second-order discrete and differential inclusions are described with endpoint constraints.

In Section 3, the discrete problem with the second-order discrete inclusions posed in Section 2 is reduced to a convex problem of a finite number of geometric constraints. We prove the necessary and sufficient conditions of optimality in terms of $L A M s$ by using constructions of convex and nonsmooth analysis.

In Section 4, we use difference approximations of derivatives and grid functions on a uniform grid to approximate problems with second-order differential inclusions. Then we derive the necessary and sufficient condition for optimality in both forms of Euler-Lagrange inclusions and transversality conditions for the discrete-approximation problem.

In Section 5, we present some applications of the results obtained for problems with second-order discrete inclusions and set-valued mappings.

\section{Needed facts and problem statement}

For the convenience of the reader, the books Mahmudov [1] and Mordukhovich [2] contain all the necessary notions and results from set-valued 
analysis theory. Let $\mathbb{R}^{n}$ be an $n$-dimensional Euclidean space, $\langle x, v\rangle$ be an inner product of elements $x, v \in \mathbb{R}^{n},(x, v)$ be a pair of $x, v$. Assume that $F: \mathbb{R}^{n} \times \mathbb{R}^{n} \rightrightarrows \mathbb{R}^{n}$ is a set-valued mapping from $\mathbb{R}^{2 n}=\mathbb{R}^{n} \times \mathbb{R}^{n}$ into the set of subsets of $\mathbb{R}^{n}$. The set-valued mapping $F$ is said to be convex if its graph $g p h F=\left\{\left(x, v_{1}, v_{2}\right): v_{2} \in F\left(x, v_{1}\right)\right\}$ is a convex subset of $\mathbb{R}^{3 n}$. A set-valued mapping $F$ is convex-closed if its $g p h F$ is a convexclosed set in $\mathbb{R}^{3 n}$. It is convex-valued if $F\left(x, v_{1}\right)$ is a convex set for each $\left(x, v_{1}\right) \in \operatorname{dom} F$, where $\operatorname{dom} F=\left\{\left(x, v_{1}\right): F\left(x, v_{1}\right) \neq \emptyset\right\}$.

Let us introduce the Hamiltonian function and argmaximum set for a set-valued mapping $F$ as

$$
\begin{gathered}
H_{F}\left(x, v_{1}, v_{2}^{*}\right)=\sup _{v_{2}}\left\{\left\langle v_{2}, v_{2}^{*}\right\rangle: v_{2} \in F\left(x, v_{1}\right)\right\} \\
F\left(x, v_{1} ; v_{2}^{*}\right)=\left\{v_{2} \in F\left(x, v_{1}\right):\left\langle v_{2}, v_{2}^{*}\right\rangle\right. \\
\left.=H_{F}\left(x, v_{1}, v_{2}^{*}\right)\right\}
\end{gathered}
$$

$v_{2}^{*} \in \mathbb{R}^{n}$, respectively. For convex $F$, we set $H_{F}\left(x, v_{1}, v_{2}^{*}\right)=-\infty$ if $F\left(x, v_{1}\right)=\emptyset$. Clearly, the Hamiltonian function $H_{F}\left(\cdot, \cdot, v_{2}^{*}\right)$ is concave for the convex set-valued mapping $F$.

Definition 1. The convex cone $K_{A}\left(z_{0}\right), z_{0}=$ $\left(x_{0}, u_{0}, v_{0}\right)$ is called the cone of tangent directions at a point $z_{0} \in A$ to the set $A$ if from $\bar{z}=(\bar{x}, \bar{u}, \bar{v}) \in K_{A}\left(z_{0}\right)$ it follows that $\bar{z}$ is a tangent vector to the set $A$ at point $z_{0} \in A$, i.e., there exists such function $\gamma(\lambda) \in \mathbb{R}^{3 n}$ such that $z_{0}+\lambda \bar{z}+\gamma(\lambda) \in A$ for sufficiently small $\lambda>0$ and $\lambda^{-1} \gamma(\lambda) \rightarrow 0$ as $\lambda \downarrow 0$.

It should be pointed out that the cone $K_{A}\left(z_{0}\right)$ is not uniquely defined. Since $\lambda \bar{z}, \lambda \geq 0$ is a vector of tangent direction, if $\bar{z}$ is the same, then it is clear that such vectors form a cone. In any case we can see that the wider a cone of tangent directions we have the essentially necessary condition for a minimum [1].

Obviously, for a convex mapping $F$ at a point $\left(x^{0}, v_{1}^{0}, v_{2}^{0}\right) \in g p h F$ setting $\gamma(\lambda) \equiv 0$, we have

$$
\begin{gathered}
K_{g p h F}\left(x^{0}, v_{1}^{0}, v_{2}^{0}\right)=\text { cone }\left[g p h F-\left(x^{0}, v_{1}^{0}, v_{2}^{0}\right)\right] \\
=\left\{\left(\bar{x}, \overline{v_{1}}, \overline{v_{2}}\right): \bar{x}=\lambda\left(x-x^{0}\right), \overline{v_{1}}=\lambda\left(v_{1}-v_{1}^{0}\right),\right. \\
\left.\overline{v_{2}}=\lambda\left(v_{2}-v_{2}^{0}\right)\right\}, \forall\left(x, v_{1}, v_{2}\right) \in g p h F .
\end{gathered}
$$

For a convex mapping $F$ a set-valued function defined by

$$
\begin{gathered}
F^{*}\left(v_{2}^{*} ;\left(x^{0}, v_{1}^{0}, v_{2}^{0}\right)\right):=\left\{\left(x^{*}, v_{1}^{*}\right):\left(x^{*}, v_{1}^{*},-v_{2}^{*}\right)\right. \\
\left.\in K_{g p h F}^{*}\left(x^{0}, v_{1}^{0}, v_{2}^{0}\right)\right\}
\end{gathered}
$$

is a locally adjoint set-valued mapping ( $L A M)$ to $F$ at a point $\left(x^{0}, v_{1}^{0}, v_{2}^{0}\right) \in g p h F$, where
$K_{g p h F}^{*}\left(x^{0}, v_{1}^{0}, v_{2}^{0}\right)$ is the dual to the cone of tangent vectors $K_{g p h F}\left(x^{0}, v_{1}^{0}, v_{2}^{0}\right)$.

Let int $A$ be the interior of the set $A \subset \mathbb{R}^{3 n}$ and riA be the relative interior of the set $A$, i.e. the set of interior points of $A$ with respect to its affine hull $A f f A$. A function $\varphi$ is called a proper function, if it does not assume the value $-\infty$ and is not identically equal to $+\infty$. Obviously $\varphi$ is proper function if and only if $\operatorname{dom} \varphi \neq \emptyset$ and $\varphi(x, y)$ is finite for $(x, y) \in \operatorname{dom} \varphi$.

We note that a convex function is continuous on the relative interior of its domain, it may have discontinuities only on its relative boundary (see, for example, Theorem 1.18 [1]).

In this paper, we study the following main secondorder discrete model:

$$
\begin{aligned}
& \text { infimum } \varphi\left(x_{N-1}, x_{N}\right), \\
& x_{t+2} \in F\left(x_{t}, x_{t+1}, t\right), t=0, \ldots, N-2, \\
& x_{0}=\alpha_{0}, x_{1}=\alpha_{1}, \\
& x_{t} \in A, t=0, \ldots, N, x_{N} \in B,
\end{aligned}
$$

where $x_{t} \in \mathbb{R}^{n}, F(\cdot, \cdot, t): \mathbb{R}^{2 n} \rightrightarrows \mathbb{R}^{n}$ is a time dependent set-valued mapping, $\varphi: \mathbb{R}^{2 n} \rightarrow \mathbb{R}$ is continuous function, $\alpha_{0}, \alpha_{1}$ are fixed vectors and $N$ is fixed natural number, $A, B \subset \mathbb{R}^{n}$ are nonempty subsets and $A \cap B \neq \emptyset$. We label this problem as $\left(P_{D}\right)$.

A sequence $\left\{x_{t}\right\}_{t=0}^{N}=\left\{x_{t}: t=0,1, \ldots, N\right\}$ is called a feasible trajectory for the stated problem $\left(P_{D}\right)$. The problem is to find a solution $\left\{\widetilde{x}_{t}\right\}_{t=0}^{N}$ of the problem $\left(P_{D}\right)$ for the second-order discrete inclusions satisfying (2) - (3) and minimizing the Mayer functional $\varphi\left(x_{N-1}, x_{N}\right)$.

The problem $\left(P_{D}\right)$ is said to be convex, if the $F(\cdot, \cdot, t), \varphi$ and $A, B$ are convex set-valued mapping, proper convex function, and convex sets respectively.

Definition 2. We say that for the convex problem $\left(P_{D}\right)$ satisfies the regularity condition, if for points $x_{t} \in \mathbb{R}^{n}$, one of the following cases is fulfilled:

(i) $\left(x_{t}, x_{t+1}, x_{t+2}\right) \in \operatorname{rigph} F(\cdot, \cdot, t), \quad t=$ $0, \ldots, N-2, x_{t} \in \operatorname{riA}, t=0, \ldots, N$, $x_{N} \in \operatorname{riB},\left(x_{N-1}, x_{N}\right) \in \operatorname{ridom} \varphi$,

(ii) $\left(x_{t}, x_{t+1}, x_{t+2}\right) \in \operatorname{intgph} F(\cdot, \cdot, t), t=$ $0, \ldots, N-2, x_{t} \in$ int $A ; x_{N} \in$ int $B$, (with the possible exception of one fixed $t$ ) and $\varphi$ is continuous at $\left(x_{N-1}, x_{N}\right)$.

It follows from the regularity condition that, if $\left\{\widetilde{x}_{t}\right\}_{t=0}^{N}$ is the optimal trajectory in the problem $\left(P_{D}\right)$, then the cones of tangent directions $K_{g p h F(\cdot, t)}\left(\tilde{x}_{t}, \tilde{x}_{t+1}, \tilde{x}_{t+2}\right)$ are not separable and 
consequently the condition of Theorem 3.2 [1] is satisfied.

We are interested in the approximate problem for the following evolution differential inclusions with endpoint constraint

$$
\begin{aligned}
& \text { infimum } \phi\left(x(1), x^{\prime}(1)\right), \\
& x^{\prime \prime}(t) \in F\left(x(t), x^{\prime}(t), t\right), \text { a.e. } t \in[0,1], \\
& x\left(0=\beta_{0}, x^{\prime}(0)=\beta_{1},\right. \\
& x(t) \in A, t \in[0,1], x(1) \in B,
\end{aligned}
$$

where $F(\cdot, \cdot, t): \mathbb{R}^{2 n} \rightrightarrows \mathbb{R}^{n}$ is a time dependent set-valued mapping, $\phi: \mathbb{R}^{2 n} \rightarrow \mathbb{R}$ is continuous function and $\beta_{0}, \beta_{1}$ are fixed vectors and $A, B \subset \mathbb{R}^{n}$ are nonempty subsets and $A \cap B \neq \emptyset$. That problem is called the viability problem.

The problem is to find an arc $\widetilde{x}(\cdot)$ of the viability problem satisfying (4) almost everywhere on $[0,1]$ and the initial and endpoint constraints that minimizes the cost functional $\phi$. Here a feasible trajectory $x(\cdot)$ is understood to be an absolutely continuous function on a time interval $[0,1]$ together with the first-order derivatives for which $x^{\prime \prime}(\cdot) \in L_{1}^{n}([0,1])$. Clearly, such a class of functions is a Banach space, endowed with the different equivalent norms.

Clearly, the condition (2) in the main discrete model is a discrete analog of second-order differential inclusions (44).

Definition 3. We say $F(\cdot, \cdot, t)$ is viable if for every $x(0)=\beta_{0}, x^{\prime}(0)=\beta_{1}$, (4) has an absolutely continuous solution $x(\cdot):[0,1] \rightarrow \mathbb{R}^{n}$ such that $x(t) \in A$ for all $t \in[0,1]$ and the inclusion in (4) is satisfied for almost everywhere on $[0,1]$.

Let us formulate the conditions of optimality for the discrete problem $\left(P_{D}\right)$ before we begin the discussion on the approximation problem with second-order inclusions.

\section{Necessary and sufficient conditions of optimality for discrete problem}

We consider the discrete problem $\left(P_{D}\right)$ in this section. First, let us introduce a vector $u=$ $\left(x_{0}, x_{1}, \ldots, x_{N}\right) \in \mathbb{R}^{n(N+1)}$ and define the following convex sets in the space $\mathbb{R}^{n(N+1)}$

$$
\begin{gathered}
M_{t}=\left\{u=\left(x_{0}, \ldots, x_{N}\right):\left(x_{t}, x_{t+1}, x_{t+2}\right)\right. \\
\in \operatorname{gph} F(\cdot, \cdot, t)\}, t=0,1, \ldots, N-2, \\
P_{t}=\left\{u=\left(x_{0}, \ldots, x_{N}\right): x_{t} \in A\right\}, t=0, \ldots, N, \\
Q=\left\{u=\left(x_{0}, \ldots, x_{N}\right): x_{N} \in B\right\}, \\
Q_{0}=\left\{u=\left(x_{0}, \ldots, x_{N}\right): x_{0}=\alpha_{0}\right\},
\end{gathered}
$$

$$
Q_{1}=\left\{u=\left(x_{0}, \ldots, x_{N}\right): x_{1}=\alpha_{1}\right\} .
$$

The discrete problem $\left(P_{D}\right)$ is now reduced to solving a convex programming problem by setting $f(u)=\varphi\left(x_{N-1}, x_{N}\right)$. In fact, it is easy to see that the problem $\left(P_{D}\right)$ is equivalent to the following one:

$$
\text { minimize } f(u)
$$

$u \in C:=\left(\bigcap_{t=0}^{N-2} M_{t}\right) \cap\left(\bigcap_{t=0}^{N} P_{t}\right) \cap Q \cap Q_{0} \cap Q_{1}$, where $C$ is the convex set. This transformation allows us to prove rigorously that if $\left\{\widetilde{x}_{t}\right\}_{t=0}^{N}$ is the optimal solution to the problem $\left(P_{D}\right)$, then $\widetilde{u}$ is the solution to the problem (5). It should be noted that under the regularity condition Definition 2 the dual cone associated with intersection of cones of tangent directions is equal to the algebraic sum of their dual cones. Thus, from Theorem 1.11 [1], we have

$$
\begin{gathered}
K_{C}^{*}(\widetilde{u})=\sum_{t=0}^{N-2} K_{M_{t}}^{*}(\widetilde{u})+\sum_{t=0}^{N} K_{P_{t}}^{*}(\widetilde{u})+K_{Q}^{*}(\widetilde{u}) \\
+K_{Q_{0}}^{*}(\widetilde{u})+K_{Q_{1}}^{*}(\widetilde{u}) .
\end{gathered}
$$

Moreover, the results taken from [1] provide necessary conditions of optimality for the convex mathematical programming (5). We can prove necessary conditions of optimality for problem (5) with geometric constraints based on results concerning convex mathematical programming. Thus, by continuity of $\varphi$ at points of some feasible solution $\left\{\widetilde{x}_{t}\right\}_{t=0}^{N}$ it follows from Theorem 3.4 [1], there exist vectors $u^{*}(t) \in K_{M_{t}}^{*}(\tilde{u}), t=$ $0,1, \ldots, N-2, w^{*}(t) \in K_{P_{t}}^{*}(\tilde{u}), t=0, \ldots, N$ and $v^{*}(0) \in K_{Q_{0}}^{*}(\tilde{u}), v^{*}(1) \in K_{Q_{1}}^{*}(\tilde{u}), v^{*}(N) \in K_{Q}^{*}(\tilde{u})$ and the number $\mu \in\{0,1\}$, not all equal to zero, such that

$$
\begin{gathered}
\mu \hat{u}^{*}=\sum_{t=0}^{N-2} u^{*}(t)+\sum_{t=0}^{N} w^{*}(t)+v^{*}(N) \\
+v^{*}(0)+v^{*}(1),
\end{gathered}
$$

where $\hat{u}^{*} \in \partial_{u} f(\tilde{u})$. From definition of the function $f$ it is easy to see that the vector $\hat{u}^{*} \in$ $\partial_{u} f(\tilde{u})$ has a form $\hat{u}^{*}=(\underbrace{0, \ldots, 0}_{N-1}, \hat{x}_{N-1}^{*}, \hat{x}_{N}^{*})$, $\left(\hat{x}_{N-1}^{*}, \hat{x}_{N}^{*}\right) \in \partial_{\left(x, v_{1}\right)} \varphi\left(\hat{x}_{N-1}, \hat{x}_{N}\right)$. First of all it is not hard to compute the dual cones $K_{P_{t}}^{*}(\widetilde{u})$, $K_{Q}^{*}(\widetilde{u}), K_{Q_{0}}^{*}(\widetilde{u})$ and $K_{Q_{1}}^{*}(\widetilde{u})$ as follows

$$
\begin{gathered}
K_{P_{t}}^{*}(\widetilde{u})=\left\{w^{*}(t): w_{t}^{*}(t) \in K_{A}^{*}\left(\widetilde{x}_{t}\right), w_{k}^{*}(t)=0,\right. \\
k \neq t\}, t=0, \ldots, N \\
K_{Q}^{*}(\widetilde{u})=\left\{v^{*}(N): v_{k}^{*}(N)=0, k \neq N\right\}, \\
K_{Q_{0}}^{*}(\widetilde{u})=\left\{v^{*}(0): v_{k}^{*}(0)=0, k \neq 0\right\}, \\
K_{Q_{1}}^{*}(\widetilde{u})=\left\{v^{*}(1): v_{k}^{*}(1)=0, k \neq 1\right\} .
\end{gathered}
$$


Then we should compute the dual cone $K_{M_{t}}^{*}(\widetilde{u})$ in the following lemma.

Lemma 1. Let $K_{g p h F}\left(x_{t}, x_{t+1}, x_{t+2}\right)$, $\left(x_{t}, x_{t+1}, x_{t+2}\right) \in g p h F$ be cone of tangent directions. Then

$$
\begin{gathered}
K_{M_{t}}^{*}(u)=\left\{u^{*}=\left(x_{0}^{*}, \ldots, x_{N}^{*}\right):\left(x_{t}^{*}, x_{t+1}^{*}, x_{t+2}^{*}\right)\right. \\
\in K_{g p h F}^{*}\left(x_{t}, x_{t+1}, x_{t+2}\right), \\
\left.x_{k}^{*}=0, k \neq t, t+1, t+2\right\} .
\end{gathered}
$$

Proof. From the dual cone definition, $u^{*} \in K_{M_{t}}^{*}(u)$ is valid if and only if $\left\langle u^{*}, \bar{u}\right\rangle=\sum_{k=0}^{N}\left\langle x_{k}^{*}, \bar{x}_{k}\right\rangle \geq 0, \forall \bar{u} \in K_{M_{t}}(u)$. Clearly, $K_{M_{t}}(u)=\left\{\bar{u}:\left(\bar{x}_{t}, \bar{x}_{t+1}, \bar{x}_{t+2}\right) \in\right.$ $\left.K_{g p h F}\left(x_{t}, x_{t+1}, x_{t+2}\right)\right\}$ and from the arbitrariness of components $\bar{x}_{k}, k \neq t, t+1, t+2$ of vectors $\bar{u}$ it follows that $x_{k}^{*}=0, k \neq t, t+1, t+2$. Therefore the inequality $\left\langle x_{t}^{*}, \bar{x}_{t}\right\rangle+\left\langle x_{t+1}^{*}, \bar{x}_{t+1}\right\rangle+$ $\left\langle x_{t+2}^{*}, \bar{x}_{t+2}\right\rangle \geq 0$ yields $\left(x_{t}^{*}, x_{t+1}^{*}, x_{t+2}^{*}\right) \in$ $K_{g p h F}^{*}\left(x_{t}, x_{t+1}, x_{t+2}\right)$.

Now, we are ready to give the conditions of optimality for the discrete problem $\left(P_{D}\right)$.

Theorem 1. Let $F(\cdot, \cdot, t)$ be a convex set-valued mapping and $\varphi$ be proper convex functional and continuous at the points of some feasible trajectory. Then for optimality of the trajectory $\left\{\tilde{x}_{t}\right\}_{t=0}^{N}$ in the Mayer problem (11) - (3) with second-order discrete inclusions, initial and endpoint constraints, it is necessary that there exist a number $\mu \in\{0,1\}$ and vectors $x_{t}^{*}, \xi_{t}^{*}, \eta_{t}^{*}, t=0, \ldots, N$ not all equal zero satisfying the Euler-Lagrange discrete inclusions and transversality conditions:

(i) $\left(x_{t}^{*}-\xi_{t}^{*}-\eta_{t}^{*}, \xi_{t+1}^{*}\right) \in F^{*}\left(x_{t+2}^{*} ;\left(\tilde{x}_{t}, \tilde{x}_{t+1}, \tilde{x}_{t+2}\right), t\right)$,

$$
t=0,1, \ldots, N-2 \text {, }
$$

(ii) $\eta_{t}^{*} \in K_{A}^{*}\left(\tilde{x}_{t}\right) ; t=0, \ldots, N, \xi_{N}^{*} \in K_{B}^{*}\left(\tilde{x}_{N}\right)$,

(iii) $\left(\xi_{N-1}^{*}-x_{N-1}^{*}+\eta_{N-1}^{*}, \xi_{N}^{*}+\eta_{N}^{*}-x_{N}^{*}\right)$

$$
\in \mu \partial_{\left(x, v_{1}\right)} \varphi\left(\tilde{x}_{N-1}, \tilde{x}_{N}\right) \text {. }
$$

In addition, under the regularity condition these conditions are sufficient for optimality of the trajectory $\left\{\tilde{x}_{t}\right\}_{t=0}^{N}$.

Proof. Obviously, (5) is a convex problem with geometric constraints and by hypotheses of the theorem, $\tilde{u}=\left(\tilde{x}_{0}, \ldots, \tilde{x}_{N}\right)$ is a solution of the problem (5). According to Lemma 10 one has

$u^{*}(t)=\left(0, \ldots, 0, x_{t}^{*}(t), x_{t+1}^{*}(t), x_{t+2}^{*}(t), 0, \ldots, 0\right)$,

where

$$
\begin{gathered}
\left(x_{t}^{*}(t), x_{t+1}^{*}(t), x_{t+2}^{*}(t)\right) \in K_{g p h F}^{*}\left(\tilde{x}_{t}, \tilde{x}_{t+1}, \tilde{x}_{t+2}\right), \\
t=0,1, \ldots, N-2,
\end{gathered}
$$

$$
\begin{gathered}
v^{*}(0)=\left(v_{0}^{*}(0), 0, \ldots, 0\right), \\
v^{*}(1)=\left(0, v_{1}^{*}(1), 0 \ldots, 0\right), \\
v^{*}(N)=\left(0,0 \ldots, 0, v_{N}^{*}(N)\right) .
\end{gathered}
$$

Now using the component-wise representation of (7), we deduce that

$$
\begin{gathered}
x_{0}^{*}(0)+w_{0}^{*}(0)+v_{0}^{*}(0)=0, \\
x_{1}^{*}(0)+x_{1}^{*}(1)+w_{1}^{*}(1)+v_{1}^{*}(1)=0, \\
x_{t}^{*}(t)+x_{t}^{*}(t-1)+x_{t}^{*}(t-2)+w_{t}^{*}(t)=0, \\
t=2, \ldots, N-2,
\end{gathered}
$$

$x_{N-1}^{*}(N-2)+x_{N-1}^{*}(N-3)+w_{N-1}^{*}(N-1)=\mu \hat{x}_{N-1}^{*}$, $x_{N}^{*}(N-2)+w_{N}^{*}(N)+v_{N}^{*}(N)=\mu \hat{x}_{N}^{*}$,

$\left(\hat{x}_{N-1}^{*}, \hat{x}_{N}^{*}\right) \in \partial_{\left(x, v_{1}\right)} \varphi\left(\hat{x}_{N-1}, \hat{x}_{N}\right), \mu \in\{0,1\}$.

On the other hand, by definition of $L A M$ we derive that

$$
\begin{aligned}
\left(x_{t}^{*}(t), x_{t+1}^{*}(t)\right) & \in F^{*}\left(-x_{t+2}^{*}(t) ;\left(\tilde{x}_{t}, \tilde{x}_{t+1}, \tilde{x}_{t+2}\right), t\right), \\
t & =2, \ldots, N-2 .
\end{aligned}
$$

Introducing the new notations $-x_{t+2}^{*}(t) \equiv x_{t+2}^{*}$, $x_{t+1}^{*}(t) \equiv \xi_{t+1}^{*}, t=0, \ldots, N-2$ and $w_{t}^{*}(t) \equiv \eta_{t}^{*}$, $t=0, \ldots, N, v_{N}^{*}(N) \equiv \xi_{N}^{*}$, we find from the formulas (9) and (10) that

$$
\begin{gathered}
\left(x_{t}^{*}-\xi_{t}^{*}-\eta_{t}^{*}, \xi_{t+1}^{*}\right) \in F^{*}\left(x_{t+2}^{*} ;\left(\tilde{x}_{t}, \tilde{x}_{t+1}, \tilde{x}_{t+2}\right), t\right), \\
t=2, \ldots, N-2,
\end{gathered}
$$

and

$$
\eta_{t}^{*} \in K_{A}^{*}\left(\tilde{x}_{t}\right) ; t=0, \ldots, N, \xi_{N}^{*} \in K_{B}^{*}\left(\tilde{x}_{N}\right) .
$$

Moreover, it is easy to see that setting, $v_{0}^{*}(0)=$ $\xi_{0}^{*}-x_{0}^{*}$ and $v_{1}^{*}(1)=-x_{1}^{*}$ in the first relationships of (8), we can generalize the formula (11) to the cases $t=0,1$. Finally, for $t=N-1$ and $t=N$ we have $\mu \hat{x}_{N-1}^{*}=-x_{N-1}^{*}+\xi_{N-1}^{*}+\eta_{N-1}^{*}$, $\mu \hat{x}_{N}^{*}=-x_{N}^{*}+\xi_{N}^{*}+\eta_{N}^{*}$ which imply

$$
\begin{gathered}
\left(-x_{N-1}^{*}+\xi_{N-1}^{*}+\eta_{N-1}^{*},-x_{N}^{*}+\xi_{N}^{*}+\eta_{N}^{*}\right) \\
\in \mu \partial_{\left(x, v_{1}\right)} \varphi\left(\tilde{x}_{N-1}, \tilde{x}_{N}\right) .
\end{gathered}
$$

Thus taking into account the formulas (11), (12) and (13), we complete the first part of the proof of theorem. The proof of the sufficiency conditions, is based on the Theorem 1.30 [1], under the regularity condition, the representation (13) holds with parameter $\mu=1$ for the point $\hat{u}^{*} \in \partial_{u} f(\tilde{u}) \cap K_{C}^{*}(\tilde{u})$.

\section{The conditions of optimality for discrete approximation problem}

In what follows, we give an idea of the construction of the discrete-approximation problem for the 
viability problem (4) with second-order differential inclusions by introducing the first and secondorder difference operators:

$$
\begin{gathered}
\Delta x(t)=\frac{1}{\delta}(x(t+\delta)-x(t)), \\
\Delta^{2} x(t)=\frac{1}{\delta}(\Delta x(t+\delta)-\Delta x(t)), t=0, \delta, \ldots 1-\delta,
\end{gathered}
$$

where $\delta$ is a step on the $t$-axis and $x(t) \equiv x_{\delta}(t)$ is a grid functions on a uniform grid on $[0,1]$. We define the following discrete-approximation problem associated with the continuous problem (4)

$$
\begin{aligned}
& \operatorname{minimize} \phi_{0}(x(1-\delta), \Delta x(1-\delta)), \\
& \Delta^{2} x(t) \in F(x(t), \Delta x(t), t), \\
& t=0, \delta, 2 \delta, \ldots, 1-2 \delta, \\
& x(0)=\beta_{0}, \Delta x(0)=\beta_{1}, \\
& x(t) \in A, t=\delta, \ldots, 1, x(1) \in B .
\end{aligned}
$$

We apply the results of Theorem 1 to the problem (14) - (16). To do this we rewrite the discreteapproximation inclusion (15) in the relevant form by introducing the following auxiliary set-valued mapping

$$
G\left(x, v_{1}, t\right):=2 v_{1}-x+\delta^{2} F\left(x, \frac{v_{1}-x}{\delta}, t\right) .
$$

Then we rewrite the problem (14) - (16) as follows

$$
\begin{aligned}
& \operatorname{minimize} \varphi_{0}(x(1-\delta), x(1)), \\
& x(t+2 \delta) \in G(x(t), x(t+\delta), t), \\
& x(0)=\beta_{0}, \Delta x(0)=\beta_{1}, \\
& x(t) \in A, t=\delta, \ldots, 1, x(1) \in B,
\end{aligned}
$$

where $\phi_{0}(x(1-\delta), \Delta x(1-\delta)) \equiv \varphi_{0}(x(1-\delta), x(1))$. By Theorem 1 for optimality of the trajectory $\{\tilde{x}(t)\}:=\{\tilde{x}(t): t=0, \delta, \ldots, 1\}$ in the problem, (18) - (19) it is necessary that there exist vectors $\bar{x}^{*}(t), \bar{\xi}^{*}(t), \bar{\eta}^{*}(t)$ and a number $\mu=\mu_{\delta} \in\{0,1\}$, not all zero, such that

$$
\begin{gathered}
\left(\bar{x}^{*}(t)-\bar{\eta}^{*}(t)-\bar{\xi}^{*}(t), \bar{\xi}^{*}(t+\delta)\right) \\
\in G^{*}\left(\bar{x}^{*}(t+2 \delta) ;(\tilde{x}(t), \tilde{x}(t+\delta), \tilde{x}(t+2 \delta)), t\right), \\
t=0, \ldots, 1-2 \delta \\
\bar{\eta}^{*}(t) \in K_{A}^{*}(\tilde{x}(t)), \bar{\xi}^{*}(1) \in K_{B}^{*}(\tilde{x}(1)), \\
\left(\bar{\eta}^{*}(1-\delta)+\bar{\xi}^{*}(1-\delta)-\bar{x}^{*}(1-\delta),\right. \\
\left.\bar{\eta}^{*}(1)+\bar{\xi}^{*}(1)-\bar{x}^{*}(1)\right) \\
\in \mu \partial_{\left(x, v_{1}\right)} \varphi_{0}(\tilde{x}(1-\delta), \tilde{x}(1)) .
\end{gathered}
$$

We should express the $L A M G^{*}$ in the above relationship (20) in terms of $L A M F^{*}$, which plays a central role in our developments in the next results.
Usually, some equivalence theorems are needed for any development in problems given by differential inclusions. Let us first prove two propositions concerning the Hamiltonian functions of the setvalued mappings $F$ and $G$, and the sets of subdifferential of the Hamiltonian functions $H_{G}$ and $H_{F}$.

Lemma 2. Let $F$ and $G$ be formula-specified convex set-valued mappings (17). Then there is the following relation between the Hamiltonian $H_{G}$ and $H_{F}$ functions:

$H_{G}\left(x, v_{1}, v_{2}^{*}\right)=\left\langle 2 v_{1}-x, v_{2}^{*}\right\rangle+\delta^{2} H_{F}\left(x, \frac{v_{1}-x}{\delta}, v_{2}^{*}\right)$.

Proof. We get the lemma proof immediately as follows, keeping in mind the definition of the Hamiltonian functions of the set-valued mappings $G, F$

$$
\begin{gathered}
H_{G}\left(x, v_{1}, v_{2}^{*}\right)=\sup \left\{\left\langle v_{2}, v_{2}^{*}\right\rangle: v_{2} \in G\left(x, v_{1}\right)\right\} \\
=\left\langle 2 v_{1}-x, v_{2}^{*}\right\rangle+\delta^{2} \sup \left\{\left\langle v_{3}, v_{2}^{*}\right\rangle: v_{3} \in F\left(x, \frac{v_{1}-x}{\delta}\right)\right\} \\
=\left\langle 2 v_{1}-x, v_{2}^{*}\right\rangle+\delta^{2} H_{F}\left(x, \frac{v_{1}-x}{\delta}, v_{2}^{*}\right) .
\end{gathered}
$$

Lemma 3. The following relation holds for subdifferentials of the Hamiltonian functions $H_{G}$ and $H_{F}$ :

$$
\begin{gathered}
\partial H_{G}\left(x, v_{1}, v_{2}^{*}\right)=\left\{-v_{2}^{*}\right\} \times\left\{2 v_{2}^{*}\right\} \\
+\delta^{2} \Theta^{*} \partial H_{F}\left(x, \frac{v_{1}-x}{\delta}, v_{2}^{*}\right)
\end{gathered}
$$

where $\Theta=\left(\begin{array}{cc}I & 0 \\ \frac{-I}{\delta} & \frac{I}{\delta}\end{array}\right)$ is a $2 n \times 2 n$ matrix partitioned into submatrices, $I, \frac{-I}{\delta}, \frac{-I}{\delta}$ and $n \times n$ zero matrix, where $I$ is an $n \times n$ identity matrix and $\Theta^{*}$ is transposes of $\Theta$.

Proof. The subdifferential $\partial H_{F}\left(x, \frac{v_{1}-x}{\delta}, v_{2}^{*}\right)$ should be computed. Notice that Hamiltonian function is concave and it is understood as $\partial_{\left(x, v_{1}\right)} H_{G}\left(x, v_{1}, v_{2}^{*}\right)=-\partial_{\left(x, v_{1}\right)}\left[-H_{G}\left(x, v_{1}, v_{2}^{*}\right)\right]$. Let $\psi_{i}: \mathbb{R}^{2 n} \rightarrow \mathbb{R}, i=1,2$, be a convex functions at a point $\left(x, v_{1}\right)$ and $g: \mathbb{R}^{2 n} \rightarrow \mathbb{R}$ be a convex function continuous at a point $\left(\psi_{1}\left(x, v_{1}\right), \psi_{2}\left(x, v_{1}\right)\right)$. Then for subdifferential of composition $f\left(x, v_{1}\right)=g\left(\psi_{1}\left(x, v_{1}\right), \psi_{2}\left(x, v_{1}\right)\right)$ the following formula is valid

$$
\partial f\left(x, v_{1}\right)=\Theta^{*} \partial g\left(\psi_{1}\left(x, v_{1}\right), \psi_{2}\left(x, v_{1}\right)\right)
$$

where

$$
\Theta=\left(\begin{array}{ll}
\frac{\partial \psi_{1}}{\partial x} & \frac{\partial \psi_{1}}{\partial v_{1}} \\
\frac{\partial \psi_{2}}{\partial x} & \frac{\partial \psi_{2}}{\partial v_{1}}
\end{array}\right)
$$


is $2 n \times 2 n$ matrix $\frac{\partial \psi_{1}}{\partial x}, \frac{\partial \psi_{1}}{\partial v_{1}}, \frac{\partial \psi_{2}}{\partial x}, \frac{\partial \psi_{2}}{\partial v_{1}}$ are Jacobian matrices and $\Theta^{*}$ is transposes of $\Theta$. Taking $\psi_{1}\left(x, v_{1}\right) \equiv x, \psi_{2}\left(x, v_{1}\right) \equiv \frac{v_{1}-x}{\delta}$ in $\Theta$, then it is easy to compute that $\Theta=\left(\begin{array}{cc}I & 0 \\ \frac{-I}{\delta} & \frac{I}{\delta}\end{array}\right)$ where $I$ is an $n \times n$ identity matrix. Then, for fixed $v_{2}^{*}$ setting $f\left(x, v_{1}\right)=H_{F}\left(x, \frac{v_{1}-x}{\delta}, v_{2}^{*}\right)$ and using Moreau-Rockafeller Theorem 1.29 [1], Lemma 2 and the formula (23), we obtain the desired result.

In the construction of $L A M$ for the original discrete approximation problem (14)-(16), the following theorem which is definitely of independent interest plays a crucial role.

Theorem 2. If $G$ is a convex set-valued mapping defined by (17), then the following statements for the $L A M s$ are equivalent:

(a) $\left(x^{*}, v_{1}^{*}\right) \in G^{*}\left(v_{2}^{*} ;\left(x, v_{1}, v_{2}\right)\right), v_{2} \in G\left(x, v_{1} ; v_{2}^{*}\right)$,

(b) $\left(\frac{x^{*}+v_{1}^{*}-v_{2}^{*}}{\delta^{2}}, \frac{v_{1}^{*}-2 v_{2}^{*}}{\delta}\right)$

$$
\begin{gathered}
\in F^{*}\left(v_{2}^{*} ;\left(x, \frac{v_{1}-x}{\delta}, \frac{x-2 v_{1}+v_{2}}{\delta^{2}}\right)\right), \\
\frac{x-2 v_{1}+v_{2}}{\delta^{2}} \in F\left(x, \frac{v_{1}-x}{\delta} ; v_{2}^{*}\right), v_{2}^{*} \in \mathbb{R}^{n}
\end{gathered}
$$

where $G\left(x, v_{1} ; v_{2}^{*}\right)$ is the argmaximum set for mapping $G$.

Proof. By useful Frobenius formula, the following inverse of $2 \times 2$ block matrices holds if $A, B$ are invertible

$\left(\begin{array}{cc}A & D \\ C & B\end{array}\right)^{-1}=\left(\begin{array}{cc}\left(A-D B^{-1} C\right)^{-1} & -A^{-1} D \Delta^{-1} \\ -\Delta^{-1} C A^{-1} & \Delta^{-1}\end{array}\right)$

where $\Delta=B-C A^{-1} D$ is the Schur complement of $A$. Therefore, denoting $A=\delta^{2} I, B=\delta I$, $C=0, D=-\delta I$, we compute that

$$
\left(\delta^{2} \Theta^{*}\right)^{-1}=\left(\begin{array}{cc}
\delta^{2} I & -\delta I \\
0 & \delta I
\end{array}\right)^{-1}=\left(\begin{array}{cc}
\frac{I}{\delta^{2}} & \frac{I}{\delta^{2}} \\
0 & \frac{I}{\delta}
\end{array}\right) .
$$

Furthermore, by Lemma 3 it is easy to see that $\left(x^{*}, v_{1}^{*}\right) \in \partial_{\left(x, v_{1}\right)} H_{G}\left(x, v_{1}, v_{2}^{*}\right)$ and

$$
\begin{aligned}
& \left(\delta^{2} \Theta^{*}\right)^{-1}\left(x^{*}+v_{2}^{*}, v_{1}^{*}-2 v_{2}^{*}\right) \\
& \in \partial_{\left(x, v_{1}\right)} H_{F}\left(x, \frac{v_{1}-x}{\delta}, v_{2}^{*}\right)
\end{aligned}
$$

are equivalent. Then from (24) and (25), it means that $\left(x^{*}, v_{1}^{*}\right) \in \partial_{\left(x, v_{1}\right)} H_{G}\left(x, v_{1}, v_{2}^{*}\right)$ if and only if

$$
\begin{array}{r}
\left(\frac{x^{*}+v_{1}^{*}-v_{2}^{*}}{\delta^{2}}, \frac{v_{1}^{*}-2 v_{2}^{*}}{\delta}\right) \\
\in \partial_{\left(x, v_{1}\right)} H_{F}\left(x, \frac{v_{1}-x}{\delta}, v^{*}\right) .
\end{array}
$$

Since $F^{*}\left(v_{2}^{*} ;\left(x, v_{1}, v_{2}\right)\right)=\partial_{\left(x, v_{1}\right)} H_{F}\left(x, v_{1}, v_{2}^{*}\right)$, $v_{2} \in F\left(x, v_{1} ; v_{2}^{*}\right)$ and by using the fact that $\partial_{\left(x, v_{1}\right)} H_{F}\left(x, v_{1}, v_{2}^{*}\right)=-\partial_{\left(x, v_{1}\right)}\left(-H_{F}\left(x, v_{1}, v_{2}^{*}\right)\right)$, we express (26) in term of $L A M s$. Here take into account that $v_{2} \in G\left(x, v_{1} ; v_{2}^{*}\right)$ and $\frac{x-2 v_{1}+v_{2}}{\delta^{2}} \in F\left(x, \frac{v_{1}-x}{\delta} ; v_{2}^{*}\right)$ ensure that the $L A M s$ are nonempty at a given points.

Lemma 4. It turns out that the following inclusions are equivalent:

$$
\begin{aligned}
& \text { (1) }\left(\bar{x}^{*}, \bar{y}^{*}\right) \in \partial \varphi_{0}\left(z_{0}\right), \\
& \text { (2) }\left(\bar{x}^{*}+\bar{y}^{*}, \delta \bar{y}^{*}\right) \in \partial \phi_{0}\left(w_{0}\right) .
\end{aligned}
$$

where $z_{0}=\left(x^{0}, y^{0}\right) \in \operatorname{dom} \varphi_{0}$ and $w_{0}=$ $\left(x^{0}, \frac{y^{0}-x^{0}}{\delta}\right) \in \operatorname{dom} \phi_{0}$.

Proof. By the definition of subdifferential sets we can write

$$
\begin{gathered}
\partial \varphi_{0}\left(z_{0}\right)=\left\{\left(\bar{x}^{*}, \bar{y}^{*}\right): \varphi_{0}(x, y)-\varphi_{0}\left(x^{0}, y^{0}\right)\right. \\
\geq\left\langle\bar{x}^{*}, x-x^{0}\right\rangle+\left\langle\bar{y}^{*}, y-y^{0}\right\rangle \\
\left.\forall(x, y) \in \mathbb{R}^{2 n}\right\} . \\
\partial \phi_{0}\left(w_{0}\right)=\left\{\left(x^{*}, y^{*}\right): \phi_{0}(w)-\varphi_{0}\left(w_{0}\right)\right. \\
\geq\left\langle x^{*}, x-x^{0}\right\rangle+\left\langle y^{*}, \frac{y-x}{\delta}-\frac{y^{0}-x^{0}}{\delta}\right\rangle \\
\left.\forall w=\left(x, \frac{y-x}{\delta}\right) \in \mathbb{R}^{2 n}\right\} .
\end{gathered}
$$

This latter relationship gives that

$$
\begin{gathered}
\partial \phi_{0}\left(w_{0}\right)=\left\{\left(x^{*}, y^{*}\right): \varphi_{0}(x, y)-\varphi_{0}\left(x^{0}, y^{0}\right)\right. \\
\geq\left\langle x^{*}-\frac{y^{*}}{\delta}, x-x^{0}\right\rangle+\left\langle\frac{y^{*}}{\delta}, y-y^{0}\right\rangle \\
\left.\forall w \in \mathbb{R}^{2 n}\right\} .
\end{gathered}
$$

When (27) and (28) are compared, we deduce that

$$
\bar{x}^{*}=x^{*}-\frac{y^{*}}{\delta}, \quad \bar{y}^{*}=\frac{y^{*}}{\delta}
$$

or, in other words,

$$
x^{*}=\bar{x}^{*}+\bar{y}^{*}, \quad y^{*}=\delta \bar{y}^{*} .
$$

The proof of theorem is completed.

Theorem 3. Let $F$ be a convex set-valued mapping and $\phi_{0}$ be proper convex functional and continuous at the points of some feasible trajectory. Then for optimality of the trajectory $\{\tilde{x}(t)\}$ in the discrete approximation problem, it is necessary that there exist a number $\mu=\mu_{\delta} \in\{0,1\}$ and vectors $x^{*}(t), \eta^{*}(t), v^{*}(t)$ which are not all equal zero, satisfying the approximate Euler-Lagrange and transversality inclusions:

$$
\begin{gathered}
(a)\left(\Delta^{2} x^{*}(t)+\Delta v^{*}(t)-\eta^{*}(t), v^{*}(t+\delta)\right) \\
\in F^{*}\left(x^{*}(t+2 \delta) ;\left(\tilde{x}(t), \Delta \tilde{x}(t), \Delta^{2} \tilde{x}(t), t\right)\right), \\
t=0, \delta, \ldots, 1-2 \delta,
\end{gathered}
$$

(b) $\eta^{*}(t) \in K_{A}^{*}(\tilde{x}(t)), \xi^{*}(1) \in K_{A \cap B}^{*}(\tilde{x}(1))$, 
(c) $\left(v^{*}(1-\delta)+\Delta x^{*}(1-\delta)+\xi^{*}(1)\right.$

$$
\begin{aligned}
& \left.+\delta \eta^{*}(1-\delta), \delta \xi^{*}(1)-x^{*}(1)\right) \\
& \in \mu \partial \phi_{0}(\tilde{x}(1-\delta), \Delta \tilde{x}(1-\delta)) .
\end{aligned}
$$

And, under the regularity condition these conditions are also sufficient for optimality of the trajectory $\{\tilde{x}(t)\}$.

Proof. By taking into account the condition (20) and by Theorem 2, it can be shown that

$$
\begin{gathered}
\left(\frac{\bar{x}^{*}(t)-\bar{\eta}^{*}(t)-\bar{\xi}^{*}(t)+\bar{\xi}^{*}(t+\delta)-x^{*}(t+2 \delta)}{\delta^{2}},\right. \\
\left.\frac{\bar{\xi}^{*}(t+\delta)-2 x^{*}(t+2 \delta)}{\delta}\right) \\
\in F^{*}\left(\bar{x}^{*}(t+2 \delta) ;\left(\tilde{x}(t), \Delta \tilde{x}(t), \Delta^{2} \tilde{x}(t), t\right)\right), \\
t=0, \ldots, 1-2 \delta .
\end{gathered}
$$

Denoting $\bar{v}^{*}(t)=\frac{\bar{\xi}^{*}(t)-2 x^{*}(t+\delta)}{\delta}$, we get

$$
\begin{gathered}
\left(\Delta^{2} \bar{x}^{*}(t)+\Delta \bar{v}^{*}(t)-\frac{\bar{\eta}^{*}(t)}{\delta^{2}}, \bar{v}^{*}(t+\delta)\right) \\
\in F^{*}\left(\bar{x}^{*}(t+2 \delta) ;\left(\tilde{x}(t), \Delta \tilde{x}(t), \Delta^{2} \tilde{x}(t), t\right)\right), \\
t=0, \ldots, 1-2 \delta .
\end{gathered}
$$

Now observe that $L A M F^{*}$ is positive homogeneous on the first argument and setting $\delta \bar{x}^{*}(t)$, $\delta \bar{v}^{*}(t)$ and $\bar{\eta}^{*}(t)$ are denoted by $x^{*}(t), v^{*}(t)$ and $\delta \eta^{*}(t)$, respectively, we derive the approximate Euler-Lagrange inclusion of theorem. Moreover, by the formula (22) and Lemma 4, we have

$$
\begin{gathered}
\left(\bar{\eta}^{*}(1-\delta)+\bar{\xi}^{*}(1-\delta)-\bar{x}^{*}(1-\delta)+\bar{\eta}^{*}(1)+\bar{\xi}^{*}(1)-\bar{x}^{*}(1),\right. \\
\left.\delta\left(\bar{\eta}^{*}(1)+\bar{\xi}^{*}(1)-\bar{x}^{*}(1)\right)\right) \\
\in \mu \partial \phi_{0}(\tilde{x}(1-\delta), \Delta \tilde{x}(1-\delta)) .
\end{gathered}
$$

Then setting $\bar{\xi}^{*}(1)=\xi^{*}(1)-\delta \eta^{*}(1)$, we find that the transversality condition of theorem:

$$
\begin{gathered}
\left(v^{*}(1-\delta)+\Delta x^{*}(1-\delta)+\xi^{*}(1)+\delta \eta^{*}(1-\delta),\right. \\
\left.\delta \xi^{*}(1)-x^{*}(1)\right) \\
\in \mu \partial \phi_{0}(\tilde{x}(1-\delta), \Delta \tilde{x}(1-\delta)) .
\end{gathered}
$$

By the condition (21), we have $\delta \eta^{*}(t) \in K_{A}^{*}(\widetilde{x}(t))$ and $\xi^{*}(1)-\delta \eta^{*}(1) \in K_{B}^{*}(\widetilde{x}(1))$. Then $\xi^{*}(1) \in$ $K_{A}^{*}(\widetilde{x}(1))+K_{B}^{*}(\widetilde{x}(1))=K_{A \cap B}^{*}(\widetilde{x}(1))$ which completes the proof of theorem.

Remark 1. For future directions, we note that the results obtained in this section could be useful for deriving optimality conditions for the secondorder viability problem (4) given by differential inclusions with endpoint constraint. At least we emphasize that the key to our success is to pass formally to the limit in the conditions of Theorem 3. Consequently, by setting $\mu=1$ and passing formally to the limit as $\delta \rightarrow 0$, the sufficient conditions of optimality can be formulated for the continuous problem (4). Moreover, by using the functional analysis approach in the convex problem, the necessity of these conditions for optimality can be justified.

Corollary 1. Let $F$ be a convex set-valued mapping and $\phi$ be proper convex functional and continuous at the points of some feasible trajectory. Then for optimality of the trajectory $\tilde{x}(t)$ in the problem (4), it is sufficient that there exist a number $\mu$ and vectors $x^{*}(t), \eta^{*}(t), v^{*}(t)$ which are not all equal zero, satisfying the second-order EulerLagrange type adjoint

$$
\begin{gathered}
(i)\left(x^{*^{\prime \prime}}(t)+v^{*^{\prime}}(t)-\eta^{*}(t), v^{*}(t)\right) \\
\in F^{*}\left(x^{*}(t) ;\left(\tilde{x}(t), \tilde{x}^{\prime}(t), \tilde{x}^{\prime \prime}(t), t\right)\right), t \in[0,1] \\
(i i) \quad \tilde{x}^{\prime \prime}(t) \in F\left(x(t), x^{\prime}(t) ; x^{*}(t), t\right), \text { a.e. } t \in[0,1], \\
\eta^{*}(t) \in K_{A}^{*}(\tilde{x}(t)), t \in[0,1),
\end{gathered}
$$

and the transversality conditions at the endpoint $t=1$ consist of the following

$$
\begin{gathered}
(i i i)\left(v^{*}(1)+x^{*^{\prime}}(1)+\xi^{*}(1),-x^{*}(1)\right) \\
\in \mu \partial \phi\left(\tilde{x}(1), \tilde{x}^{\prime}(1)\right), \\
\xi^{*}(1) \in K_{A \cap B}^{*}(\tilde{x}(1)) .
\end{gathered}
$$

Note that the transformation to the continuous problem (4) is in any case, a separate subject of discussion and is therefore omitted. Note also that this construction of optimality conditions in the presented paper for second-order discrete and discrete approximation inclusions can be useful for the optimization of an arbitrary order discrete and ordinary differential inclusions.

\section{Applications}

In this section, we describe some interesting applications of the problem (11)-(3). At first, let us consider the problem with the "linear discrete" structure

$$
\begin{gathered}
\text { minimum } \varphi\left(x_{N-1}, x_{N}\right), \\
x_{t+2} \in F\left(x_{t}, x_{t+1}, t\right), t=0, \ldots, N-2, \\
F\left(x, v_{1}\right)=C_{0} x+C_{1} v_{1}+D u, u \in U \\
x_{0}=\alpha_{0}, x_{1}=\alpha_{1}, \\
x_{t} \in A, t=0, \ldots, N, x_{N} \in B,
\end{gathered}
$$

where $C_{0}, C_{1}$ are $n \times n, D$ is $n \times r$ matrix, $D \subset \mathbb{R}^{r}$ is convex closed set, $\varphi$ is continuously differentiable function. It is required to find controlling parameters $\widetilde{u}_{t} \in U(t=0, \ldots, N)$ such that the corresponding trajectory $\left\{\widetilde{x}_{t}\right\}_{t=0}^{N}$ minimizes $\varphi$. We observe that in this case $F\left(x, v_{1}\right)=\left\{v_{2}=\right.$ $\left.C_{0} x+C_{1} v_{1}+D u: u \in U\right\}$. It is not hard to 
compute that

$$
\begin{gathered}
F^{*}\left(v_{2}^{*} ;\left(x, v_{1}, v_{2}\right)\right) \\
= \begin{cases}\left(C_{0}^{*} v_{2}^{*}, C_{1}^{*} v_{2}^{*}\right), & -D^{*} v_{2}^{*} \in K_{U}^{*}(u), \\
\emptyset, & -D^{*} v_{2}^{*} \notin K_{U}^{*}(u),\end{cases}
\end{gathered}
$$

where $v_{2}=C_{0} x+C_{1} v_{1}+D u, u \in U, C_{0}^{*}, C_{1}^{*}$ and $D^{*}$ are transposed matrices. Then by using Theorem 1 and from the formula (30), we have $x_{t}^{*}-\xi_{t}^{*}-\eta_{t}^{*}=C_{0}^{*} x_{t+2}^{*}$ and $\xi_{t+1}^{*}=C_{1}^{*} x_{t+2}^{*}, t=$ $0, \ldots, N-2$ and $-D^{*} x_{t+2}^{*} \in K_{U}^{*}(u), \eta_{t}^{*} \in K_{A}^{*}\left(\widetilde{x}_{t}\right)$ and $\xi_{N}^{*} \in K_{B}^{*}\left(\widetilde{x}_{N}\right)$. Clearly the transversality conditions consist of the following:

$$
\begin{gathered}
\xi_{N-1}^{*}-x_{N-1}^{*}+\eta_{N-1}^{*}=\mu \varphi_{x} \\
\xi_{N}^{*}+\eta_{N}^{*}-x_{N}^{*}=\mu \varphi_{v_{1}} .
\end{gathered}
$$

Moreover $-D^{*} v_{2}^{*} \in K_{U}^{*}(u)$ means that the Weierstrass Pontryagin maximum condition

$$
\left\langle D \widetilde{u}_{t}, x_{t}^{*}\right\rangle=\sup _{u \in U}\left\langle D u, x_{t}^{*}\right\rangle
$$

is satisfied. The regularity condition is superfluous for linear problems. It is, therefore, necessary and sufficient for the optimality of the trajectory $\left\{x_{t}\right\}_{t=0}^{N}$ in linear problem that there exists $\left\{x_{t}^{*}\right\}_{t=0}^{N}$ satisfying the second-order Euler-Lagrange differential equation with $\mu=1$ and Weierstrass Pontryagin maximum principle (31).

Now, let us consider another example where a setvalued mapping is defined by nonlinear inequality:

$$
\begin{gathered}
\text { minimum } \varphi\left(x_{N-1}, x_{N}\right), \\
x_{t+2} \in F\left(x_{t}, x_{t+1}, t\right), t=0, \ldots, N-2, \\
F\left(x, v_{1}\right)=\left\{v_{2}: \Psi\left(x, v_{1}, v_{2}\right) \leq 0\right\} \\
x_{0}=\alpha_{0}, x_{1}=\alpha_{1} \\
x_{t} \in A, t=0, \ldots, N, x_{N} \in B
\end{gathered}
$$

where the function $\Psi$ and $\varphi$ are continuously differentiable functions. Then according to the Theorem 2.13. [1] by elementary computations we find that

$$
\begin{gathered}
F^{*}\left(v_{2}^{*} ;\left(x, v_{1}, v_{2}\right)\right)=\left\{\left(-\mu \Psi_{x}^{\prime}\left(x, v_{1}, v_{2}\right),\right.\right. \\
\left.-\mu \Psi_{v_{1}}^{\prime}\left(x, v_{1}, v_{2}\right)\right): v_{2}^{*}=\mu \Psi_{v_{2}}^{\prime}\left(x, v_{1}, v_{2}\right), \\
\left.\mu \Psi\left(x, v_{1}, v_{2}\right)=0, \mu \geq 0,\right\}
\end{gathered}
$$

where $\Psi_{x}^{\prime}\left(x, v_{1}, v_{2}\right), \Psi_{v_{1}}^{\prime}\left(x, v_{1}, v_{2}\right)$ and $\Psi_{v_{2}}^{\prime}\left(x, v_{1}, v_{2}\right)$ are gradient vectors with respect to $x, v_{1}, v_{2}$, correspondingly. So by using Theorem 1, we get the following relations

$$
\begin{gathered}
x_{t}^{*}-\xi_{t}^{*}-\eta_{t}^{*}=-\mu_{t} \Psi_{x}^{\prime}\left(\widetilde{x}_{t}, \widetilde{x}_{t+1}, \widetilde{x}_{t+2}\right), \\
\xi_{t+1}^{*}=-\mu_{t} \Psi_{v_{1}}^{\prime}\left(\widetilde{x}_{t}, \widetilde{x}_{t+1}, \widetilde{x}_{t+2}\right), \\
x_{t+2}^{*}=\mu_{t} \Psi_{v_{2}}^{\prime}\left(\widetilde{x}_{t}, \widetilde{x}_{t+1}, \widetilde{x}_{t+2}\right), \\
\mu_{t} \Psi\left(\widetilde{x}(t), \widetilde{x}_{t+1}, \widetilde{x}_{t+2}\right)=0, \\
\mu_{t} \geq 0, t=0, \ldots, N-2,
\end{gathered}
$$

$$
\eta_{t}^{*} \in K_{A}^{*}\left(\widetilde{x}_{t}\right), t=0, \ldots, N, \xi_{N}^{*} \in K_{B}^{*}\left(\widetilde{x}_{N}\right),
$$

and transversality conditions

$$
\begin{gathered}
\xi_{N-1}^{*}-x_{N-1}^{*}+\eta_{N-1}^{*}=\varphi_{x}\left(\widetilde{x}_{N-1}, \widetilde{x}_{N}\right) \\
\xi_{N}^{*}+\eta_{N}^{*}-x_{N}^{*}=\varphi_{v_{1}}\left(\widetilde{x}_{N-1}, \widetilde{x}_{N}\right) .
\end{gathered}
$$

So we have proved the following theorem.

Theorem 4. If $\Psi$ and $\varphi$ are continuously differential functions and the cone $K_{g p h F}\left(x, v_{1}, v_{2}\right)$ is non-empty for all $\left(\widetilde{x}_{t}, \widetilde{x}_{t+1}, \widetilde{x}_{t+2}\right)$ then the existence of $\left\{x_{t}^{*}, \xi_{t}^{*}, \eta_{t}^{*}\right\}$ satisfying the adjoint discrete inclusions (34)-(36) is necessary and sufficient for the optimality of the trajectory $\left\{\widetilde{x}_{t}\right\}_{t=0}^{N}$ of problem (32).

Acknowledgments. The author wishes to express her sincere thanks to anonymous reviewers for valuable suggestions which improved the final manuscript.

\section{References}

[1] Mahmudov, E.N. (2011). Approximation and optimization of discrete and differential inclusions. Elsevier, Boston, USA.

[2] Mordukhovich, B.S. (2006). Variational analysis and generalized differentiation, $I: B a-$ sic theory; II: Applications, Grundlehren Series (Fundamental Principles of Mathematical Sciences), Vol. 330 and 331, Springer.

[3] Bohner, M., Ding, Y., \& Došly, O. (2015). Difference equations, discrete dynamical systems and applications. Springer, Switzerland.

[4] Mahmudov, E.N. (2014). Approximation and optimization of higher order discrete and differential inclusions. Nonlin. Differ. Equat. Appl., 21, 1-26.

[5] Mahmudov, E.N. (1991). A two-parameter optimal control problem for systems of discrete inclusions. Automat. Remote Control, 52(3), 353-362.

[6] Mahmudov, E.N., \& Mardanov, M.J. (2020). On duality in optimal control problems with second-order differential inclusions and initial-point constraints. Proceed. Institute Math. Mech. Nat. Acad. Sci. Azerb., 46(1), 115-128.

[7] Özdemir, N. \& Evirgen, F. (2010). A dynamic system approach to quadratic programming problems with penalty method. Bulletin of the Malaysian Mathematical Sciences Society. Second Series, 33(1), 79-91.

[8] Ulus, A.Y. (2018). On discrete time infinite horizon optimal growth problem. An International Journal of Optimization and Control: Theories \& Applications (IJOCTA), 8(1), 102-116. 
[9] Mahmudov, E.N. (2015). Optimization of second order discrete approximation inclusions. Numeric. Funct. Anal. Optim., 36, 624-643.

[10] Mahmudov, E.N. (2018). Optimization of Mayer problem with Sturm-Liouville-type differential inclusions. J. Optim. Theory Appl., 177, 345-375.

[11] Mahmudov, N.I., Vijayakumar, V., \& Murugesu, R. (2016). Approximate controllability of second-order evolution differential inclusions in Hilbert spaces. Mediterr. J. Math., $13,3433-3454$.

[12] Auslender, A., \& Mechler, J. (1994). Second order viability problems for differential inclusions. J. Math. Anal. Appl., 181, 205-218.

[13] Veliov, V. (1992). Second-order discrete approximation to linear differential inclusions. SIAM Journal on Numerical Analysis, 29(2), 439-451.

[14] Donchev, T., Farkhi, E., \& Mordukhovich, B.S. (2007). Discrete approximations, relaxation, and optimization of one-sided Lipschitzian differential inclusions in Hilbert spaces. Journal of Differential Equations, 243(2), 301-328.

[15] Agarwal, R.P., \& O'Regan, D. (2002). Fixedpoint theory for weakly sequentially uppersemicontinuous maps with applications to differential inclusions. Nonlinear Oscillat., 5(3), 277-286.
[16] Boltyanskii, V.G. (1978). Optimal control of discrete systems. John Wiley, New York, USA.

[17] Haddad, T., \& Yarou, M. (2006). Existence of solutions for nonconvex second-order differential inclusions in the infinite dimensional space. Electron. J. Differ. Equat., 2006(33), $1-8$.

[18] Marco, L., \& Murillo, J.A. (2001). Lyapunov functions for second-order differential inclusions: a viability approach. J. Math. Anal. Appl., 262(1), 339-354.

[19] Lupulescu, V. (2005). Viable solutions for second order nonconvex functional differential inclusions. Electron. J. Differ. Equat., 110, 111.

[20] Mahmudov, E.N. (2020). Optimal control of higher order differential inclusions with functional constraints. ESAIM: Control, Optimisation and Calculus of Variations, 26, 1-23.

Sevilay Demir Sağlam is currently working as a research assistant at the Department of Mathematics, Istanbul University, Istanbul, Turkey. She received M.Sc. and Ph.D. degrees from the Department of Mathematics, Istanbul University in 2012 and 2017, respectively. Her current research interests are the polyhedral optimization, control theory, duality theory and the conditions of optimality for discrete and differential inclusions given by set-valued mappings. (10) https://orcid.org/0000-0003-4615-6863

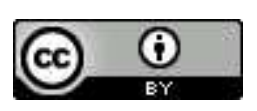

This work is licensed under a Creative Commons Attribution 4.0 International License. The authors retain ownership of the copyright for their article, but they allow anyone to download, reuse, reprint, modify, distribute, and/or copy articles in IJOCTA, so long as the original authors and source are credited. To see the complete license contents, please visit http://creativecommons.org/licenses/by/4.0/. 